\title{
Thrombocytopenia and Absent Radii (Tar) Syndrome in Non Identical Twins
}

\author{
Kage $\mathbf{A}^{1}$, Marinova $\mathbf{J}^{2}$ \\ ${ }^{1}$ Dr. Anup Kage, MBBS, MRCPCH, Paediatric Registrar, \\ ${ }^{2}$ Dr. Jasmina Marinova, MBBS, MRCPCH, Paediatric \\ Consultant. Both from the Department of Paediatrics, \\ Kettering General Hospital, Kettering, UK.
}

\section{Introduction}

T hrombocytopenia-Absent Radius (TAR) syndrome is a congenital malformation syndrome characterised by bilateral absence of the radii and a thrombocytopenia. Thrombocytopenia manifests mainly from birth but can take few weeks to manifest. Main skeletal abnormality is absent radii.

\section{The Case}

Dichorionic diamniotic twin girls were born at 37 wk gestation to healthy parents with normal phenotype. Antenatal scans showed absent radii in both the twins. Amniocentesis at the time was declined by parents. The twins were born by normal vaginal delivery in good condition with a birth weight of 2.02 and $2.3 \mathrm{~kg}$.

On examination, they had short humeri, absent radii with radial deviation of the forearms bilaterally. The thumbs were present with overlapping of the fifth over the fourth finger. They had bowed legs. Initial full blood count showed platelet count of $9000 /$ microlitre in twin one and 8000/microlitre in twin two. Both twins had a petechial rash but no active bleeding and received leucocyte depleted platelet transfusion at $20 \mathrm{ml} / \mathrm{kg}$. Cranial ultrasound scan on day two was normal. Fundoscopy showed bilateral retinal hemorrhages. Skeletal survey showed absent radii with short ulnas on both sides.. Echocardiogram on one twin was normal and showed a small ASD on the other twin.

Genetics study: Twins were confirmed to be non identical. In both twins, array CGH (Comparative Genetic Hybridization) analysis using an Agilent custom $180 \mathrm{~K}$ oligoarray showed a female array profile with three regions of imbalance - a $430 \mathrm{~Kb}$ deletion of $1 \mathrm{q} 21.1$, 2.2Mb duplication of $4 \mathrm{q} 28.3$ and a $450 \mathrm{~Kb}$ duplication

\section{Address for correspondence \\ Dr. Anup Kage \\ Email: anupkage@yahoo.co.uk}

\begin{abstract}
This is a case report of female non identical twins with bilateral absent radii and severe thrombocytopenia. Genetic studies confirmed 1q21.1 deletion. A review of literature showed TAR syndrome in 2 pairs of identical twins (reported in 1986 ) and one pair of non identical twins (reported in 1979).
\end{abstract}

Key words: Thrombocytopenia and absent radii (TAR), identical twins

of $9 q 21.11$ The $1 q 21.1$ deletion was within the region commonly deleted in TAR syndrome (OMIM 274000). There were no reports of $4 q$ and $9 q$ deletions duplication in the literature. The clinical significance of these two imbalances is unclear.

As both twins needed multiple platelet transfusions, both of them had a Hickmann line inserted. Platelets from a single donor was arranged to avoid HLA (Human Leucocyte Antigen) incompatibility. They were also reviewed by a Plastic surgeon who advised thermoplastic splints to maintain wrist neutrality and gentle stretching exercises. They propose to carry out a reconstructive surgery of wrists at one year of age.

Both the twins were on normal formula feeds after birth. They developed cow's milk intolerance soon after and were changed to extensivey hydrolysed formula milk. They developed renal tubulopathy by two months of age and needed phosphate and bicarbonate supplements. Ophthalmology review at 6 months of age showed resolved retinal hemorrhages. Primary immunisation was administered by the sub dermal route in both the twins. Parents were counseled about future pregnancies and the recurrence risk for the couple is 1 in 4 .

A review of literature showed TAR syndrome in only two pairs of identical twins ${ }^{1}$ (reported in 1986) and one fraternal twin ${ }^{2}$ (reported in 1979). There were no reports of TAR syndrome associated with renal tubulopathy.

\section{How to cite this article ?}

Kage A, Marinova J. Thrombocytopenia and Absent Radii (Tar) Syndrome in Non Identical Twins. J Nepal Paediatr Soc 2013;33(2):150-151. 


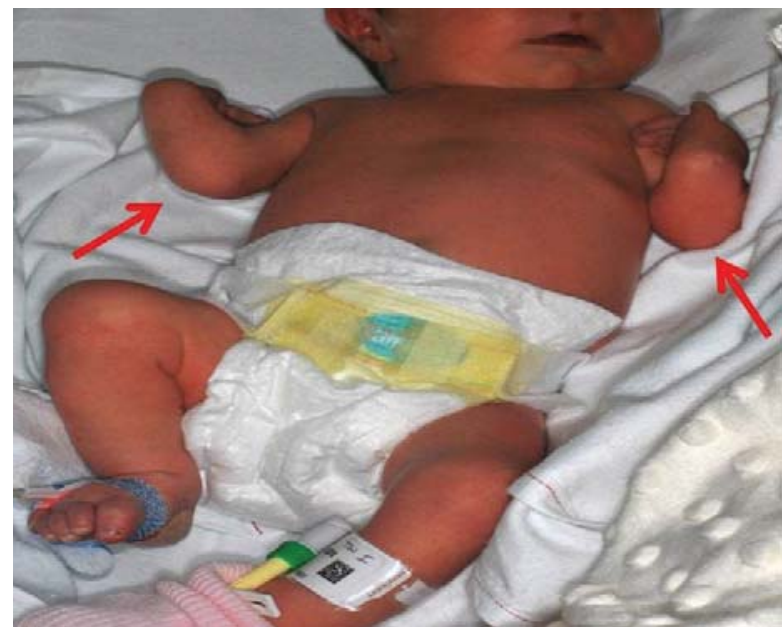

Fig 1: Showing absent radius and radial deviation of forearms bilaterally. (Twin 1)

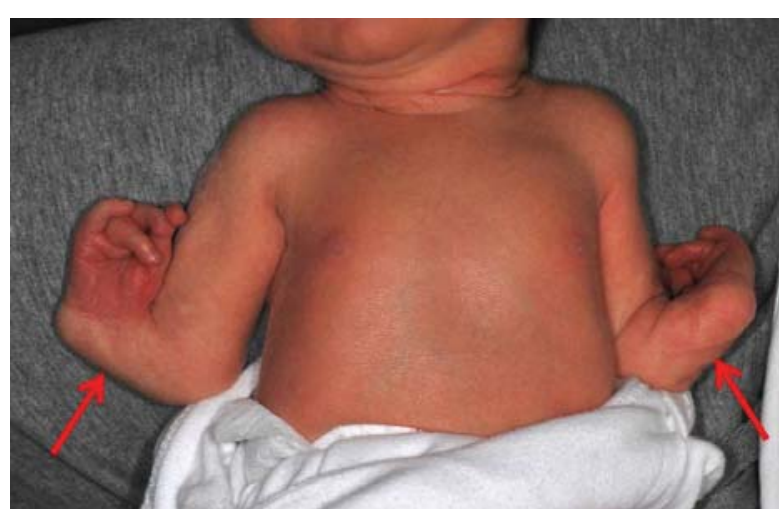

Fig 2: Showing absent radius, radial deviation of forearms and short humerus bilaterally. Thumbs are present. (Twin 2)

\section{Discussion}

TAR syndrome was first described in 1959 by Shaw and Oliver ${ }^{3}$. Hall et al ${ }^{4}$ set the diagnostic criteria and it was defined as a syndrome and classified as the association of hypomegakaryocytic thrombocytopenia and bilateral absent radii. Hematologic complications like episodic thrombocytopenia usually occurs at birth or during early infancy. Bone marrow examination reveals decreased or abnormal megakaryocytes, with normal myeloid and erythroid precursors. Bilateral absent radii is the main congenital skeletal deformity. Others include shortening and deformity of the ulnae, and occasionally absence of all the long bones in the arm. The fingers and thumbs are always present. Cardiac anomalies ${ }^{5}$, particularly the Tetralogy of Fallot and Atrial septal defects, may be present. Symptomatic cow's-milk allergy ${ }^{6}$ is associated with $47 \%$ of all cases of TAR syndrome, and patients may present as vomiting, bloody diarrhoea and failure to thrive. It can also precipitate thrombocytopenic episodes..

Neurological ${ }^{7}$ abnormalities is associated in about $7 \%$ of all cases of TAR syndrome. This association is presumed to be secondary to complications from intracranial haemorrhage precipitated by thrombocytopenia. The life expectancy is almost normal if the patient survives to two years of age ${ }^{7}$.

Occurence of genitourinary ${ }^{8}$ anomalies are rare and include horse shoe kidney, renal fused ectopia and renal stones. There are no reports of renal tubulopathy.

The differential diagnosis prior to genetic analysis would be of Robert's syndrome (hypomelia, cleft palate, microcephaly), Holt-Oram syndrome (upper limb anomalies, ASD) and Fanconi's syndrome (short stature, leg bowing, wide wrist and ankles) which can also present with a similar phenotype to TAR syndrome.

Consent: Written consent obtained from parents.

\section{References}

1. Shaw S, Oliver RAM. Congenital hypoplastic thrombocytopenia with skeletal deformities in siblings. Blood 1959;14:374-7.

2. Hall JG, Levin J, Kuhn JP, et al. Thrombocytopenia with absent radius (TAR). Medicine (Baltimore) 1969;48(6):411-39.

3. Hedberg VA, Lipton JM. Thrombocytopenia with absent radii. A review of 100 cases. Am J Pediatr Hematol Oncol 1988;10(1):51-64.

4. PF, Alder-Brecher B, Verlander PC, Pavlakis SG, Davis JG,Auerbach AD. The need for more accurate and timely diagnosis in Fanconi's anaemia - a report from the international Fanconi anaemia registry. Pediatrics 1993;91:1116-20

5. Van den Berg DJ, Franke U. Roberts syndrome: a review of 100 cases and a new scoring system for severity. Am J Med Genet 1993; 47:1104-23.

6. Newbury-Ecob RA, Leanage R, Raeburn JA, Young ID. Holt Oramsyndrome: a clinical genetic study. J Med Genet 1996; 33:306-7.

7. Hays RM, Bartoghesky LE, Feingold M. New features of thrombocytopenia and absent radius syndrome. Birth Defects 1982;18:115-121.

8. Bradshaw A, Donnelly LF, Foreman JW. Thrombocytopenia and absent radii (TAR) syndrome associated with horseshoe kidney. Pediatr Nephrol. 2000 Jan;14(1):29-31. 